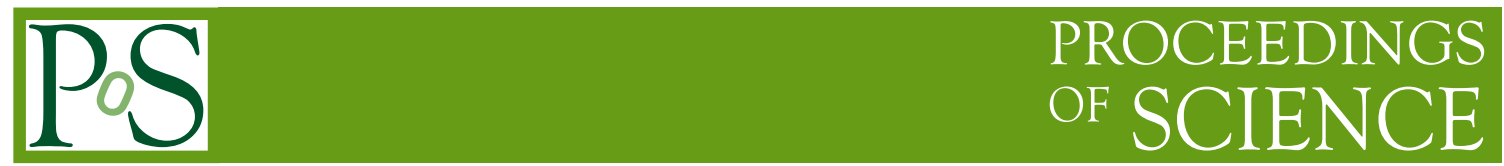

\title{
Search for Squarks and Gluinos in events with missing transverse momentum
}

\section{Robert SCHOEFBECK*广}

( Austrian Academy of Sciences Hephy Vienna ( AT))

E-mail: robert.schoefbeck@cern.ch, schoefbeckehep.itp.tuwien.ac.at

I review latest results from CMS on inclusive searches for squark and gluino production at the LHC, including third generation signatures, and present results on a variety of complementary final state signatures and methods using up to $19.4 / \mathrm{fb}$ of data from the $8 \mathrm{TeV}$ LHC run of 2012.

XXI International Workshop on Deep-Inelastic Scattering and Related Subjects 22-26 April, 2013

Marseilles, France

* Speaker.

${ }^{\dagger}$ A footnote may follow. 


\section{Introduction}

Supersymmetry (SUSY) $[1,2,3,4,5,6]$, potentially offers natural solutions to limitations of the standard model (SM), for example by canceling divergent loop effects on the Higgs boson mass. Achieving these cancellations requires that the SUSY partners of the gluon and the top quark, i.e., the gluino $(\widetilde{g})$ and the top squark $(\widetilde{t})$, have masses in the $\mathrm{TeV}$ range or below [7,8]. An extensive program of searches at the Tevatron, LEP, and LHC have found no evidence of SUSY so far and considerably constrained many SUSY models. The resulting mass limits are in the range of several hundred $\mathrm{GeV}$ for the scalar partners of the first and second generation quarks for many model scenarios. However, viable scenarios remain with $m_{\tilde{\mathrm{t}}}, m_{\tilde{\mathrm{b}}} \approx 0.5 \mathrm{TeV}$ and $m_{\tilde{g}} \approx 1 \mathrm{TeV}$. In the models considered here, the lightest supersymmetric particle (LSP) is the $\widetilde{\chi}^{0}$ which is stable and neutral.

In this note, I review latest results from CMS on inclusive searches for squark and gluino production, including third generation signatures, and present results on a variety of complementary final state signatures and methods using up to $20 \mathrm{fb}^{-1}$ of data from the $8 \mathrm{TeV}$ LHC run of 2012. Results are interpreted in the framework of Simplified Model Spectra (SMS) which are not models of fundamental interactions but theory-guided effective models which are tailored towards unambigous description of collider signatures.

\section{Search in the hadronic final states using the $\alpha_{T}$ variable}

Searches for supersymmetry in hadronic final states have to deal with large multi-jet backgrounds from standard model QCD interactions. In this search, a dimensionless kinematic variable, $\alpha_{T}$, is used as the main discriminator between events with genuine and misreconstructed missing transverse energy $\left(E_{\mathrm{T}}^{\mathrm{miss}}\right)$. The search is performed in a signal region that is binned in the number of jets $\left(n_{\text {jets }}\right)$, the scalar sum of the transverse energy of these jets $\left(H_{\mathrm{T}}\right)$, and the number of jets identified as originating from a bottom quark $\left(n_{b-\text { jets }}\right)[10]$.

The method used to estimate the (non-multijet) standard model background contributions in the signal region relies on measurements made in three kinematically similar data control samples, and the use of "translation factors" obtained from simulation. Signal region and data control samples are binned in the same way as the signal regions and the first $11.7 \mathrm{fb}^{-1}$ of the $8 \mathrm{TeV}$ dataset are used for this search.

Systematic uncertainties are assigned to the translation factors obtained from simulation, which account for theoretical uncertainties and limitations in the simulation modelling of kinematics and instrumental effects. The magnitudes of these systematic uncertainties are determined from data with a representative set of closure tests, in which yields from one of the three control samples, along with the corresponding translation factors obtained from simulation, are used to predict the yields in another control sample. The samples are selected to ensure that potential signal contamination in a wide variety of SUSY models, including those considered in this analysis, are negligible. Therefore, the closure tests carried out both between and within control samples probe the properties of the relevant SM backgrounds. In particular, the closure tests address the modelling in simulation of the relative contributions of the main SM backgrounds ( $W+$ jets, $\mathrm{t} \overline{\mathrm{t}}$ and $Z+\mathrm{jets}$ ), the kinematics of important observables such as $\alpha_{T}$, and the reconstruction of b-quark jets. 


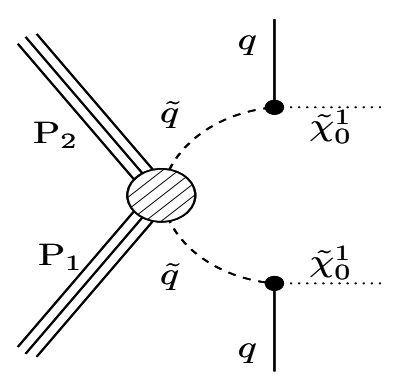

(a)

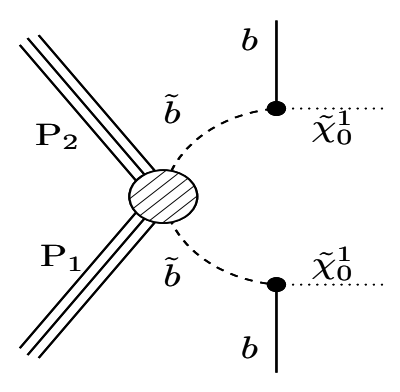

(c)

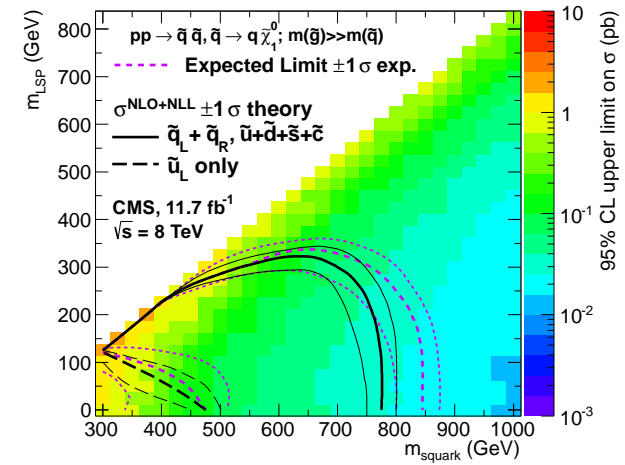

(b)

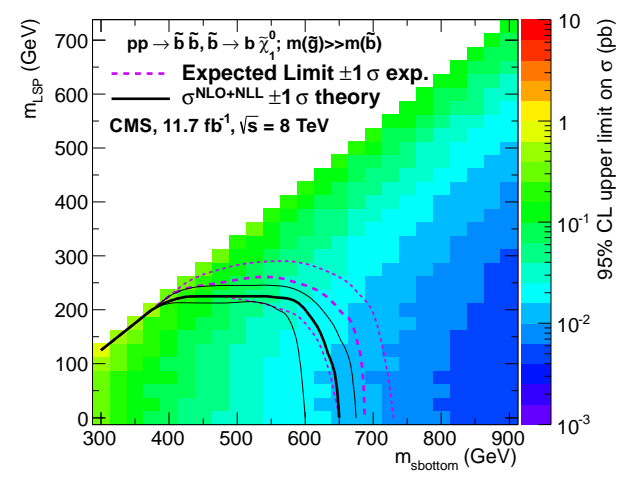

(d)

Figure 1: Model topology and exclusion cross-section versus model spectrum for pair-produced squarks, each decaying to a quark and a neutralino LSP. The two sets of exclusion contours correspond to the production of eight first- and second-generation squarks with degenerate masses or only a single light squark $(\mathrm{a}, \mathrm{b})$. Model topology and exclusion cross-section versus model spectrum for pair-produced bottom squarks, each decaying to a bottom quark and a neutralino LSP (c,d).

The search regions are defined by three different bins of jet multiplicity $\left(n_{\text {jets }}=2, n_{\text {jets }}=3\right.$ and $\left.n_{\text {jets }} \geq 4\right)$, five bins in $n_{b-\text { jets }}(=0,=1,=2,=3, \geq 4)$ and eight bins in $H_{\mathrm{T}}$ with lower bin boundaries from $275 \mathrm{GeV}$ to $875 \mathrm{GeV}$. From these regions and the modelling of all background uncertainties, a likelihood function is constructed and used to simultanously test the compatibility of the observed yield with the SM hypothesis. No significant excess above the SM expectation is observed in the signal regions and the corresponding limits for squark pair production and sbottom pair production are shown in Fig. 1. If the neutralino mass is lighter than $\approx 200 \mathrm{GeV}$, the mass limits are $m_{\widetilde{q}} \lesssim 750 \mathrm{GeV}$ and $m_{\widetilde{\mathrm{b}}} \lesssim 650 \mathrm{GeV}$. The limit on gluino mediated $\widetilde{\mathrm{b}}$ production are included in the Fig. 2 (blue).

\section{Search in final states with missing transverse energy and $0,1,2$, or $\geq 3$ b jets}

An alternative search [11] uses a similar binning in $n_{\text {jets }}$ and $n_{b-\text { jets }}$, but uses search regions defined by $E_{\mathrm{T}}^{\text {miss }}$ and $H_{\mathrm{T}}$ which are divided into four bins each. The lower boundaries of the $E_{\mathrm{T}}^{\text {miss }}$ bins are 125, 150, 250 and $350 \mathrm{GeV}$. In order to supress the QCD multi-jet background, a cut 


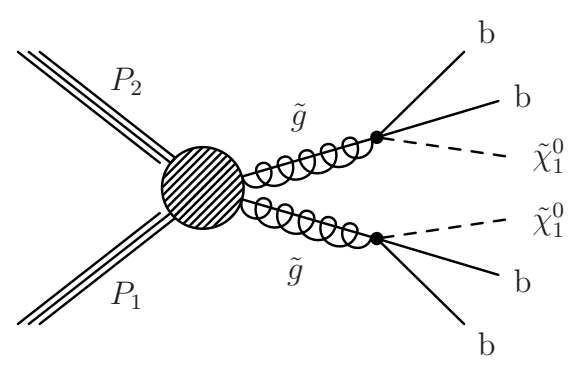

(a)

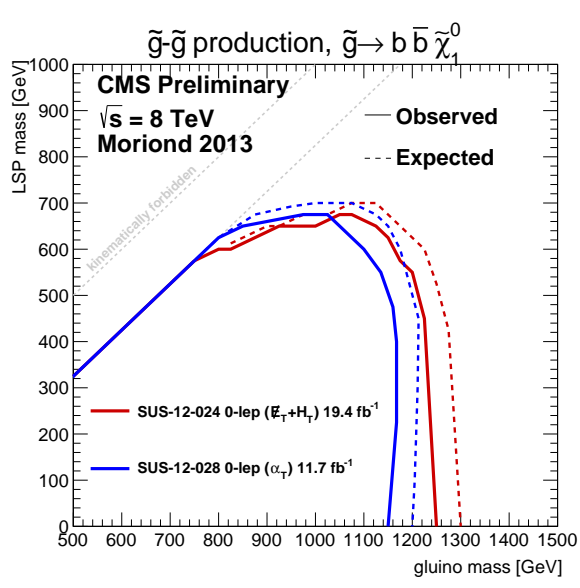

(b)

Figure 2: Model topology and summary of observed and expected limits for gluino pair production with gluino decaying via $\widetilde{g} \rightarrow \widetilde{\mathrm{b} b} \widetilde{\chi}^{0}$.

$\Delta \Phi>4$ is imposed where $\Delta \Phi=\min \left(\Delta \phi\left(\right.\right.$ jet $\left.\left._{i}, E_{\mathrm{T}}^{\text {miss }}\right) / \sigma_{i}\right)$ and $\Delta \phi\left(\right.$ jet $\left._{i}, E_{\mathrm{T}}^{\text {miss }}\right)$ is the angular distance of the $\mathrm{i}$-th jet to the $E_{\mathrm{T}}^{\text {miss }}$ vector and $\sigma_{i}$ the angular resolution of this distance. Three different data control samples are: a di-lepton sample of $\mu \mu$ and ee events in the Zmass window for extracting the $Z \rightarrow v v$ background, a 1-lepton control sample for determining the $W+$ jets and $t \bar{t}$ background and a sample with an inverted $\Delta \Phi$ cut in order to estimate the residual QCD multi-jet contribution. The translation factors take into account kinematical and acceptance differences on a bin-to-bin basis and a global scaling factor. Several closure tests on data control regions validate this procedure and are used to derive systematic uncertainties and full $8 \mathrm{TeV}$ dataset is used for this search. Finally, a global likelihood function has been constructed as a product of Poissonian PDFs, one for each bin, and from constraint PDFs for the nuisance parameters associated with the systematical uncertainties. A test statistic as described in [9] (common to all limits discussed here) is used to set limits on different SUSY models.

The limit shown in Fig. 2 assumes a $100 \%$ branching of $\widetilde{g} \rightarrow \widetilde{\mathrm{b}} \widetilde{\chi} \widetilde{\chi}^{0}$ and thus we can exclude gluino masses up to $1.25 \mathrm{TeV}$ if $m_{\tilde{\chi}^{0}} \lesssim 650 \mathrm{GeV}$. The corresponding limit on gluino mediated stop production is depicted in Fig. 4 and goes up to $1.075 \mathrm{TeV}$ for $m_{\widetilde{\chi}^{0}} \lesssim 300 \mathrm{GeV}$.

\section{The same sign di-lepton final state}

In contrast to the searches discussed so far, the search channels with same-sign, isolated leptons ( $e$ or $\mu$ ) have extremly low SM background. As a result, searches for anomalous production of same-sign dileptons do not need tight kinematical constraints on $H_{\mathrm{T}}$ and $E_{\mathrm{T}}^{\text {miss }}$ and can thus be very sensitive to new physics contributions, not limited to SUSY. Within SUSY, same-sign di-lepton events with bottom quarks can arise when the third-generation squarks are lighter than other squarks, resulting in an abundance of top and bottom quarks produced in cascade decays $[7,12,13,14,15]$. In this search, two isolated same-sign leptons with a transversal momentum exceeding $20 \mathrm{GeV}$ and consistent with originating from the same vertex are required [16]. Fur- 


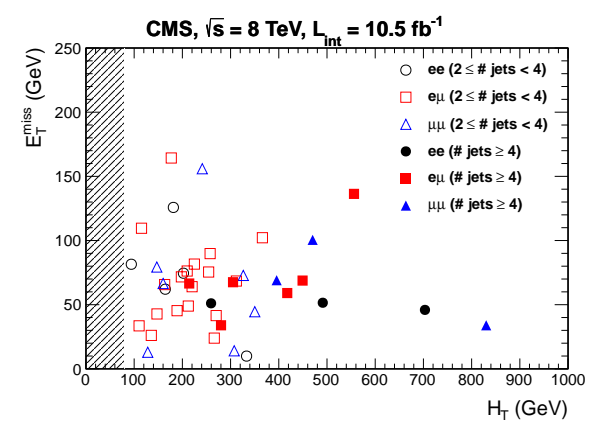

(a)

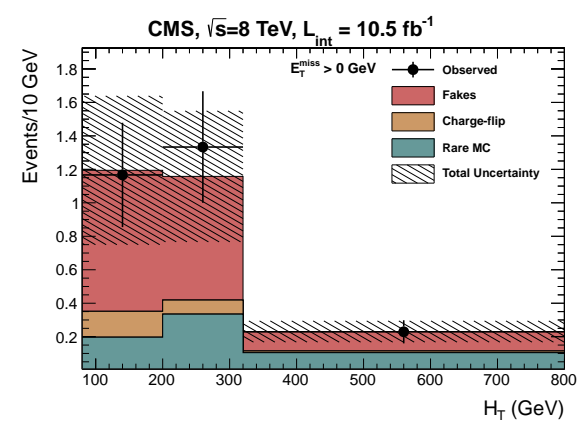

(b)

Figure 3: Distribution of $E_{\mathrm{T}}^{\text {miss }}$ vs. $H_{\mathrm{T}}$ for the 13 events when requiring $n_{b-\text { jets }} \geq 2$; ee events: circles; $e \mu$ events: squares; $\mu \mu$ events: triangles, Projection of the scatter plot above onto the $H_{\mathrm{T}}$ axis (b).

thermore, their pair invariant mass must be above $8 \mathrm{GeV}$, and at least two b-tagged jets must be reconstructed. Events are collected with dilepton triggers. Events with a third lepton are rejected if one of the opposite charge lepton pairs is consistent with a low mass di-lepton resonance or within the $Z$-mass window, $76<m_{l l}<106 \mathrm{GeV}$. The first $10.4 \mathrm{fb}^{-1}$ of the $8 \mathrm{TeV}$ dataset have been included in this analysis.

There are three sources of SM background to potential new physics signals: "fake leptons" are leptons from heavy-flavor decay, misidentified hadrons, muons from light-meson decay in flight, or electrons from unidentified photon conversions. These are estimated from a sample of events with at least one lepton that passes a loose selection but fails the full set of tight identification and isolation requirements. The background estimation uses the "tight-to-loose" ratio, i.e., the probability for a loosely identified fake lepton to also pass the full set of requirements, as determined from studies of fake leptons in multijet events. Charge flips are events with opposite-sign isolated leptons where one of the leptons is an electron and its charge is misreconstructed due to severe bremsstrahlung in the tracker material (this effect is negligible for muons). Charge flips are estimated by selecting opposite-sign $e e$ or $e \mu$ events passing the full kinematic selection, weighted by the probability of electron charge misassignment as a function of the transversal momentum and the pseudo-rapidity. This probability is obtained from $\mathrm{MC}$ simulation and is validated on a sample of $Z \rightarrow e e$ events. Finally, rare SM process such as $t \bar{t}+W / Z$ are estimated from simulation.

A total of nine signal regions in this search with different $n_{j e t}, n_{b-j e t}, E_{\mathrm{T}}^{\mathrm{miss}}$ and $H_{\mathrm{T}}$ requirements [16]. Fig. 3 shows the scatter plot of the most inclusive signal region requiring only $n_{b-j e t s} \geq 2$ and its projection onto the $H_{\mathrm{T}}$ axis. The background predictions are well in agreement with the observations. This result is used to set limits on gluino mediated $\widetilde{t}$ production, depicted in Fig. 4 (green). Gluino masses below $1 \mathrm{TeV}$ can be exluded and this limit is insensitive to $m_{\widetilde{\chi}^{0}}$ since the kinematical requirements in this search are comparably loose.

\section{Summary}

The CMS Collaboration has conducted searches for the production of Squarks and Gluinos in the $8 \mathrm{TeV}$ dataset using two different analysis in the hadronic channel and in the same sign 


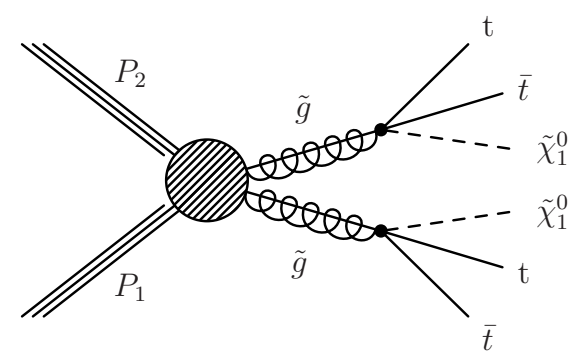

(a)

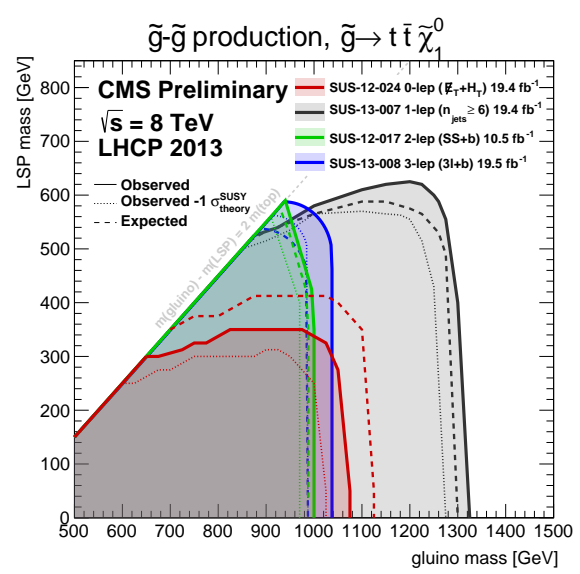

(b)

Figure 4: Model topology and summary of observed and expected limits for gluino pair production with gluino decaying via the 3-body decay into top, anti-top and neutralino.

di-lepton channel. Each analysis is binned in inclusive kinematical variables like $E_{\mathrm{T}}^{\text {miss }}$ and $H_{\mathrm{T}}$ and in multiplicities of jets and b-tagged jets. The data-driven background predictions are well in agreement with the observation and limits have been set on simplified models describing the production of squarks and gluinos, including third generation squarks.

\section{References}

[1] S. P. Martin, “A Supersymmetry primer,” In “Kane, G.L. (ed.): Perspectives on supersymmetry II” 1-153 [hep-ph/9709356].

[2] J. Wess and B. Zumino, Nucl. Phys. B 70, 39 (1974).

[3] H. P. Nilles, Phys. Rept. 110, 1 (1984).

[4] H. E. Haber and G. L. Kane, Phys. Rept. 117, 75 (1985).

[5] R. Barbieri, S. Ferrara and C. A. Savoy, Phys. Lett. B 119, 343 (1982).

[6] S. Dawson, E. Eichten and C. Quigg, Phys. Rev. D 31, 1581 (1985).

[7] M. Papucci, J. T. Ruderman and A. Weiler, JHEP 1209, 035 (2012) [arXiv:1110.6926 [hep-ph]].

[8] C. Brust, A. Katz, S. Lawrence and R. Sundrum, JHEP 1203, 103 (2012) [arXiv:1110.6670 [hep-ph]].

[9] [ATLAS Collaboration], ATL-PHYS-PUB-2011-011.

[10] S. Chatrchyan et al. [CMS Collaboration], arXiv:1303.2985 [hep-ex].

[11] S. Chatrchyan et al. [CMS Collaboration], arXiv:1305.2390 [hep-ex].

[12] A. G. Cohen, D. B. Kaplan and A. E. Nelson, Phys. Lett. B 388 (1996) 588 [hep-ph/9607394].

[13] S. Dimopoulos and G. F. Giudice, Phys. Lett. B 357, 573 (1995) [hep-ph/9507282].

[14] R. Barbieri, G. R. Dvali and L. J. Hall, Phys. Lett. B 377 (1996) 76 [hep-ph/9512388].

[15] C. Csaki, L. Randall and J. Terning, Phys. Rev. D 86 (2012) 075009 [arXiv:1201.1293 [hep-ph]].

[16] S. Chatrchyan et al. [CMS Collaboration], JHEP 1303 (2013) 037 [arXiv:1212.6194 [hep-ex]]. 